\title{
Análise do conteúdo de diagnósticos de enfermagem sobre desenvolvimento infantil
}

\author{
Juliana Martins de Souza ${ }^{1}$, \\ Maria de La Ó Ramallo Veríssimo², \\ Diná de Almeida Lopes Monteiro da Cruz ${ }^{3}$
}

\section{RESUMO}

Dispor de diagnósticos de enfermagem que respondam à complexidade do desenvolvimento infantil (DI) é fundamental para subsidiar o enfermeiro na elaboração de planos de cuidado integral à criança. $O$ objetivo deste trabalho foi apresentar a análise do conteúdo de três novos diagnósticos sobre DI para a NANDA-I. Pesquisa metodológica, de método misto. Etapa quantitativa com juízes, mediante questionário estruturado, com análise de concordância simples; e etapa qualitativa de análise das discordâncias em grupo focal com peritos, para obtenção de consenso. Dezoito juízes responderam ao questionário, com concordância acima de $80 \%$ para os diagnósticos "Atraso no Desenvolvimento Infantil" e "Risco de Atraso no Desenvolvimento Infantil"; o diagnóstico “Disposição para Desenvolvimento Infantil Melhorado" teve 61\% de concordância do enunciado e acima de 80\% na maioria das características definidoras. Oito peritos analisaram as discordâncias até produção de consensos. A elevada concordância dos juízes e consenso dos peritos validaram o conteúdo dos diagnósticos propostos.

Descritores: Desenvolvimento Infantil; Diagnóstico de Enfermagem; Estudos de Validação; Processo de Enfermagem.

\footnotetext{
${ }^{1}$ Enfermeira, Doutora em Enfermagem. Professora Adjunta da Universidade Federal de Goiás, Regional Catalão. Catalão, GO, Brasil. E-mail: jumartins2005@gmail.com.

${ }^{2}$ Enfermeira, Doutora em Enfermagem. Professora Livre-Docente da Escola de Enfermagem da Universidade de São Paulo. São Paulo, SP, Brasil. Email: mdlorver@usp.br.

${ }^{3}$ Enfermeira, Doutora em Enfermagem. Professora Titular da Escola de Enfermagem da Universidade de São Paulo. São Paulo, SP, Brasil. E-mail: dina@usp.br.
}

Artigo recebido: 23/01/2017.

Artigo aprovado: 01/12/2017.

Artigo publicado: 19/06/2018.

\section{Como citar esse artigo:}

Souza JM, Veríssimo MLOR, Cruz DALM. Análise do conteúdo de diagnósticos de enfermagem sobre desenvolvimento infantil. Rev. Eletr. Enf. [Internet]. 2018 [acesso em: ];20:v20a06. Disponível em: http://doi.org/10.5216/ree.v20.45041. 


\section{INTRODUÇÃO}

Este estudo teve como objeto a análise de conteúdo de novos diagnósticos de enfermagem relativos ao desenvolvimento infantil (DI), elaborados para a taxonomia da NANDA-International (NANDA-I), conforme descrito detalhadamente em outro texto ${ }^{(1)}$.

A promoção do desenvolvimento da criança de forma integral é reconhecida como elemento central do cuidado de enfermagem na primeira infância ${ }^{(2)}$. Assim, a existência de diagnósticos de enfermagem validados e que contemplem este fenômeno em sua complexidade é essencial para direcionar a elaboração de um plano de cuidado de enfermagem com qualidade e que responda a um dos eixos da Política Nacional de Saúde da Criança, que é a promoção do $\mathrm{DI}^{(3)}$.

Contudo, um estudo analítico demonstrou que os diagnósticos de enfermagem sobre o DI, disponíveis na taxonomia da Nanda- ${ }^{(4)}$, não contemplavam o fenômeno DI em sua especificidade e complexidade, além de apresentarem várias inconsistências para sua aplicação(5). Em seguida, um desses diagnósticos, o de "Atraso no Crescimento e Desenvolvimento", foi excluído da taxonomia ${ }^{(6)}$, com recomendação de novos estudos para abordar cada um dos conceitos em separado, confirmando-se a necessidade de sua reelaboração(5).

Buscando responder a essa lacuna, foram propostos novos diagnósticos de enfermagem relativos ao $\mathrm{DI}^{(1)}$. Tal proposição seguiu os passos recomendados para os processos de desenvolvimento de diagnósticos de enfermagem da NANDA-( ${ }^{(6-7)}$, e utilizou análise secundária dos resultados de um estudo de análise do conceito $\mathrm{DI}^{(8)}$.

A referida análise de conceito teve como referencial metodológico o modelo Híbrido ${ }^{(9)}$, e, como referencial teórico, a Teoria Bioecológica do Desenvolvimento Humano ${ }^{(10)}$.

O modelo Híbrido de análise de conceito propõe o desenvolvimento dos elementos do conceito em três fases: teórica, de campo, e analítica ${ }^{(9)}$. O uso desse modelo possibilitou a identificação dos elementos do DI, ou seja, seus antecedentes, consequentes, atributos e definições ${ }^{(8)}$.

A Teoria Bioecológica explica o desenvolvimento como fenômeno de continuidade e mudanças nas características biopsicológicas dos indivíduos ao longo do tempo, como resultado da interação de quatro elementos: processo, pessoa, contexto e tempo $(\mathrm{PPCT})^{(10)}$. O processo refere-se às interações do indivíduo com outras pessoas, objetos e símbolos em seu ambiente; o elemento pessoa contempla as características biopsicobiológicas que interferem no desenvolvimento; o elemento contexto é composto por todos os fatores do ambiente que influenciam o desenvolvimento, considerando desde o ambiente mais próximo da pessoa até o mais distante; e o elemento tempo considera o ciclo de vida humano, assim como a influência do momento histórico no desenvolvimento. Assim, o modelo PPCT destaca o efeito das interações e do ambiente em que a criança/pessoa está inserida, além dos aspectos biológicos e genéticos ${ }^{(10)}$.

A sinergia desses referenciais garantiu a elaboração de um conceito consistente e ampliado que alcançou validação por um grupo de peritos.

Posteriormente, os títulos dos diagnósticos, definições, características definidoras e fatores de risco ou fatores relacionados, foram constituídos a partir dos dados sistematizados dessa análise de conceito ${ }^{(8)}$. Portanto, os novos diagnósticos contemplaram os aspectos relativos aos processos, à pessoa/criança e aos contextos que impactam o DI ${ }^{(10)}$ conforme o modelo PPCT da Teoria Bioecológica ${ }^{(10)}$. Tais diagnósticos foram intitulados: "Atraso 
no Desenvolvimento Infantil"; "Risco de Atraso no Desenvolvimento Infantil” e "Disposição para Desenvolvimento Infantil Melhorado"(1).

O objetivo deste trabalho foi apresentar a análise do conteúdo dos componentes desses três novos diagnósticos sobre DI propostos para a taxonomia da NANDA-I, tendo em vista sua validação de conteúdo para posterior validação clínica.

\section{MÉTODO}

Estudo metodológico, aprovado pelo Comitê de Ética (CAAE: 0114.0.196.000-11) e seguiu as recomendações da Resolução 466/2012(11). A análise do conteúdo dos três novos diagnósticos revisados valeu-se de abordagem mista, com delineamento exploratório sequencial(12), entre outubro de 2013 e fevereiro de 2014. A primeira etapa foi um estudo de concordância, quantitativo, com juízes, e a segunda ocorreu em estudo de consenso, qualitativo, com peritos, como detalhado a seguir.

Para o estudo de concordância, foi elaborado um questionário para análise do título, definição, características definidoras, fatores relacionados, ou fatores de risco dos diagnósticos de enfermagem revisados. Os fatores relacionados e fatores de risco foram agrupados de acordo com as dimensões da Teoria Bioecológica do Desenvolvimento Humano(10), visando facilitar a obtenção e análise dos dados relativos ao desenvolvimento da criança durante sua avaliação.

Para cada item, havia três opções de resposta: concordo, concordo parcialmente ou discordo, além de espaço para comentários. O questionário foi apreciado e refinado em reuniões do grupo de pesquisas. Estabeleceu-se $80 \%$ como índice de concordância mínima aceitável, uma forma simples de se calcular a concordância entre os juízes ${ }^{(13)}$, sendo computadas como concordância apenas as respostas na opção "concordo".

O critério de inclusão dos juízes foi ter perfil de proficiência ${ }^{(14)}$, sendo definidos enfermeiros com titulação mínima de especialista e com experiência de pelo menos um ano na área de saúde da criança.

A coleta de dados ocorreu em um evento nacional da Sociedade Brasileira de Enfermeiros Pediatras, por favorecer o acesso a enfermeiros que atuavam em saúde da criança e atendiam aos critérios de inclusão, oriundos de várias regiões do país. O convite foi realizado durante atividades do evento. Foram distribuídos 100 questionários, juntamente com uma carta convite, uma síntese da proposta e o Termo de Consentimento Livre e Esclarecido. Adicionalmente, o convite para a pesquisa foi enviado via mensagem eletrônica para aproximadamente 40 enfermeiros cadastrados em um banco de dados do Grupo de Pesquisa Cuidado em Saúde e Promoção do Desenvolvimento Infantil (http://dgp.cnpq.br/dgp/espelhogrupo/6090275483093701).

Os resultados dos questionários foram tabulados e tratados segundo frequência simples e porcentagem, e os comentários agrupados por similaridade de conteúdo.

Para análise das discordâncias e sugestões apresentadas pelos juízes, realizou-se a segunda etapa de coleta, num grupo focal com peritos. Definiu-se como peritos para essa etapa: enfermeiros, com titulação mínima de mestre e publicações na temática, além de experiência em saúde da criança, o que os caracteriza como experts(14). O grupo foi conduzido por um coordenador, um dos pesquisadores do estudo, e as discussões foram gravadas para posterior registro detalhado. Os dados já analisados na primeira etapa foram apresentados ao grupo focal pela pesquisadora. Todos os questionamentos e sugestões dos juízes foram discutidos entre os participantes até 
obtenção de consenso. Foi considerado consenso quando a maioria do grupo concordava com a síntese da discussão sobre cada questionamento ou sugestão, realizada pelo coordenador.

\section{RESULTADOS}

\section{Caracterização dos participantes}

Participaram do estudo de concordância 18 enfermeiros, o que significou retorno de $13 \%$ dos profissionais para os quais foi divulgada a pesquisa durante o evento e por email. Estes foram oriundos de cinco estados do país, com média de idade de 36 anos, tempo de formado entre um ano a 30 anos, e apenas um respondente do sexo masculino; 14 profissionais tinham especialização em áreas diversas, 12 tinham mestrado, quatro tinham doutorado e dois cursavam residência em pediatria. Quanto às experiências em saúde da criança, 14 tinham publicações em pediatria, 17 referiram fazer avaliação do DI em sua prática profissional e 16 utilizavam diagnósticos de enfermagem na sua atuação. Quinze participantes referiram utilizar a NANDA-I na sua prática profissional.

No grupo focal, no estudo de consenso, participaram oito enfermeiros, sendo um do sexo masculino, com média de idade de 37 anos. 0 tempo de formação variou de cinco anos a 20 anos, e houve cinco mestres e três doutores. Todos atuavam com saúde da criança, realizavam avaliação de DI e utilizavam diagnósticos de enfermagem em sua prática profissional. Todos tinham publicações sobre o tema, e seis participantes atuavam em instituições de ensino.

\section{Diagnóstico de enfermagem: "Atraso no desenvolvimento infantil"}

A Tabela 1 apresenta os resultados da análise do conteúdo do diagnóstico "Atraso no desenvolvimento infantil” pelos juízes e mostra grande concordância com o novo título e definição, bem como com quase a totalidade das características definidoras e fatores relacionados propostos.

Na segunda etapa da coleta de dados, o grupo focal discutiu os elementos que não obtiveram o mínimo de $80 \%$ de concordância.

Quanto à característica definidora "Dificuldade/Incapacidade em desempenhar habilidades típicas do grupo etário", as justificativas dos juízes que não manifestaram concordância plena foram de que seria desnecessária, por estar incluída nas demais características, que abordam cada área do desenvolvimento individualmente. No entanto, na discussão do grupo, optou-se por mantê-la, uma vez que os dados que levam ao diagnóstico podem variar segundo o conteúdo do instrumento de avaliação de desenvolvimento utilizado. Assim, o instrumento de avaliação pode não especificar a área do atraso, ou, ainda, podem ser utilizados instrumentos que apresentem outras dimensões do desenvolvimento, como uma escala de desenvolvimento funcional, ou de atividades de vida diária, que trariam como resultado outras dimensões do DI que corresponderiam então a essa característica definidora mais ampla para o diagnóstico de enfermagem.

As respostas de concordância parcial com o fator relacionado "Crescimento inadequado" não foram justificadas pelos juízes. No grupo focal, chegou-se ao consenso de mantê-lo, devido ao embasamento teórico que o justifica ${ }^{(15-16)}$. 
Tabela 1: Concordância acerca dos elementos do diagnóstico Atraso no desenvolvimento infantil. São Paulo, SP, Brasil, 2014.

\begin{tabular}{|c|c|c|c|c|c|c|}
\hline \multirow[t]{2}{*}{ Elementos } & \multicolumn{2}{|c|}{ Concorda } & \multicolumn{2}{|c|}{$\begin{array}{c}\text { Concorda } \\
\text { parcialmente }\end{array}$} & \multicolumn{2}{|c|}{$\begin{array}{c}\text { Não } \\
\text { concorda }\end{array}$} \\
\hline & $\mathbf{N}$ & $\%$ & $\mathbf{N}$ & $\%$ & $\mathbf{N}$ & $\%$ \\
\hline Título: Atraso no desenvolvimento infantil & 17 & 94,4 & 01 & 5,6 & - & - \\
\hline $\begin{array}{l}\text { Definição: Criança classificada em situação de atraso no desenvolvimento } \\
\text { segundo escala de avaliação de desenvolvimento adotada como referência. }\end{array}$ & 16 & 89,9 & 02 & 11,1 & - & - \\
\hline \multicolumn{7}{|l|}{ Características definidoras } \\
\hline $\begin{array}{l}\text { Dificuldade/Incapacidade em desempenhar habilidades típicas do grupo } \\
\text { etário }\end{array}$ & 13 & 72,2 & 04 & 22,2 & 01 & 5,6 \\
\hline $\begin{array}{l}\text { Dificuldade/Incapacidade em desempenhar habilidades na linguagem típicas } \\
\text { do grupo etário }\end{array}$ & 17 & 94,4 & 01 & 5,6 & - & - \\
\hline $\begin{array}{l}\text { Dificuldade/Incapacidade em desempenhar habilidades motoras típicas do } \\
\text { grupo etário }\end{array}$ & 17 & 94,4 & 01 & 5,6 & - & - \\
\hline $\begin{array}{l}\text { Dificuldade/Incapacidade em desempenhar habilidades cognitivas típicas do } \\
\text { grupo etário }\end{array}$ & 17 & 94,4 & 01 & 5,6 & - & - \\
\hline $\begin{array}{l}\text { Dificuldade/Incapacidade em desempenhar habilidades psicossociais típicas } \\
\text { do grupo etário }\end{array}$ & 16 & 88,9 & 02 & 11 & - & - \\
\hline \multicolumn{7}{|l|}{ Fatores relacionados } \\
\hline \multicolumn{7}{|l|}{ Fatores da criança } \\
\hline Doenças & 17 & 94,4 & 01 & 5,6 & - & - \\
\hline Distúrbios genéticos & 18 & 100 & - & - & - & - \\
\hline Distúrbios congênitos & 18 & 100 & - & - & - & - \\
\hline Distúrbios sensoriais & 17 & 94,4 & - & - & 01 & 5,6 \\
\hline $\begin{array}{l}\text { Crescimento inadequado (perímetro cefálico, curvas de peso e estatura } \\
\text { muito abaixo do esperado para a idade)* }\end{array}$ & 12 & 70,6 & 04 & 23,5 & 01 & 5,9 \\
\hline Prematuridade e/ou baixo peso ao nascer* & 14 & 82,3 & 02 & 11,8 & 01 & 5,9 \\
\hline \multicolumn{7}{|l|}{ Aspectos relacionados com a gestação } \\
\hline Uso de medicações na gestação & 16 & 88,9 & 02 & 11,1 & - & - \\
\hline Uso de tabaco na gestação & 17 & 94,4 & 01 & 5,6 & - & - \\
\hline Uso de álcool na gestação & 16 & 88,9 & 02 & 11,1 & - & - \\
\hline Uso de drogas na gestação & 16 & 88,9 & 02 & 11,1 & - & - \\
\hline $\begin{array}{l}\text { Exposição a poluentes ambientais (por exemplo: dióxido de nitrogênio, } \\
\text { benzeno, chumbo, manganês, pesticida, metais pesados) }\end{array}$ & 17 & 94,4 & 01 & 5,6 & - & - \\
\hline Saúde mental materna alterada (ansiedade, depressão e estresse) & 16 & 88,9 & 02 & 11,1 & - & - \\
\hline Doença materna & 15 & 83,3 & 03 & 16,7 & - & - \\
\hline Acompanhamento pré-natal insuficiente ou ausente & 17 & 94,4 & 01 & 5,6 & - & - \\
\hline \multicolumn{7}{|l|}{ Aspectos de cuidado } \\
\hline Ausência de vínculo * & 15 & 88,2 & 02 & 11,8 & - & - \\
\hline Saúde mental materna alterada (ansiedade, depressão e estresse)* & 17 & 100 & - & - & - & - \\
\hline $\begin{array}{l}\text { Exposição à violência doméstica (negligência, maus tratos, violência } \\
\text { parental)* }\end{array}$ & 17 & 100 & - & - & - & - \\
\hline Desenvolvimento cognitivo dos pais prejudicado* & 14 & 82,3 & 03 & 17,7 & - & - \\
\hline Falta de apoio de profissional de saúde* & 15 & 88,2 & 02 & 11,8 & - & - \\
\hline Institucionalização* & 15 & 88,2 & 02 & 11,8 & - & - \\
\hline $\begin{array}{l}\text { Vivenciar situações estressantes sem ter o apoio suficiente de um cuidador } \\
\text { (adoção, entrada na creche, hospitalização, mudanças familiares)* }\end{array}$ & 16 & 94,1 & 01 & 5,9 & - & - \\
\hline $\begin{array}{l}\text { Falta de estímulo (ambiente físico inadequado, falta de oportunidade de } \\
\text { brincar, interação inconsistente do cuidador com a criança, entre outras)* }\end{array}$ & 16 & 94,1 & 01 & 5,9 & - & - \\
\hline \multicolumn{7}{|l|}{ Condições socioeconômicas } \\
\hline Economicamente desfavorecido (renda familiar insuficiente, desemprego)* & 15 & 88,2 & 02 & 11,8 & - & - \\
\hline $\begin{array}{l}\text { Condições sociais desfavoráveis (violência, falta de acesso a rede de apoio, } \\
\text { entre outras)* }\end{array}$ & 17 & 100 & - & - & - & - \\
\hline
\end{tabular}

* $\mathrm{N}$ teve valor inferior porque uma pessoa não respondeu o item. 
As sugestões de alterações nos fatores relacionados, realizadas pelos juízes, foram discutidas e aceitas no grupo, sendo elas: acrescentar a palavra "cuidador" ao fator relacionado "Ausência de vínculo", e juntar em um único fator de risco "Uso de álcool na gestação" e "Uso de drogas na gestação".

\section{Diagnóstico de enfermagem: "Risco para atraso no desenvolvimento infantil"}

A Tabela 2 apresenta os resultados da análise do conteúdo do DE “Risco para atraso no desenvolvimento infantil" pelos juízes, mostrando concordância maior que $80 \%$ para todos os elementos propostos.

Tabela 2: Concordância acerca dos elementos do diagnóstico Risco de Atraso no desenvolvimento infantil. São Paulo, SP, Brasil, 2014.

\begin{tabular}{|c|c|c|c|c|c|c|}
\hline \multirow[t]{2}{*}{ Componente } & \multicolumn{2}{|c|}{ Concorda } & \multicolumn{2}{|c|}{$\begin{array}{c}\text { Concorda } \\
\text { parcialmente }\end{array}$} & \multicolumn{2}{|c|}{$\begin{array}{l}\text { Não } \\
\text { concorda }\end{array}$} \\
\hline & $\mathbf{N}$ & $\%$ & $\mathbf{N}$ & $\%$ & $\mathbf{N}$ & $\%$ \\
\hline Título: Risco de atraso no desenvolvimento infantil & 17 & 94,4 & - & - & 01 & 5,6 \\
\hline $\begin{array}{l}\text { Definição: Risco de apresentar atraso no desenvolvimento devido exposição a } \\
\text { situações que interferem negativamente no desenvolvimento* }\end{array}$ & 15 & 88,2 & 02 & 11,8 & - & - \\
\hline \multicolumn{7}{|l|}{ Fatores relacionados } \\
\hline \multicolumn{7}{|l|}{ Fatores da criança } \\
\hline Doenças & 18 & 100 & - & - & - & - \\
\hline Distúrbios genéticos & 17 & 94,4 & 01 & 5,6 & - & - \\
\hline Distúrbios congênitos & 18 & 100 & - & - & - & - \\
\hline Distúrbios sensoriais & 18 & 100 & - & - & - & - \\
\hline $\begin{array}{l}\text { Crescimento inadequado (perímetro cefálico, curvas de peso e estatura } \\
\text { muito abaixo do esperado para a idade) }\end{array}$ & 18 & 100 & - & - & - & - \\
\hline Prematuridade e/ou baixo peso ao nascer & 18 & 100 & - & - & - & - \\
\hline $\begin{array}{l}\text { Ser classificada como risco para o desenvolvimento segundo escala de } \\
\text { avaliação padronizada }\end{array}$ & 18 & 100 & - & - & - & - \\
\hline \multicolumn{7}{|l|}{ Aspectos relacionados com a gestação } \\
\hline Uso de medicações na gestação & 17 & 94,4 & 01 & 5,6 & - & - \\
\hline Uso de tabaco na gestação & 17 & 94,4 & 01 & 5,6 & - & - \\
\hline Uso de álcool na gestação & 18 & 100 & - & - & - & - \\
\hline Uso de drogas na gestação & 16 & 88,9 & 02 & 11,1 & - & - \\
\hline $\begin{array}{l}\text { Exposição a poluentes ambientais (por exemplo: dióxido de nitrogênio, } \\
\text { benzeno, chumbo, manganês, pesticida, metais pesados) }\end{array}$ & 16 & 88,9 & 02 & 11,1 & - & - \\
\hline Saúde mental materna alterada (ansiedade, depressão e estresse) & 17 & 94,4 & 01 & 5,6 & - & - \\
\hline Doença materna & 16 & 88,9 & 02 & 11,1 & - & - \\
\hline Acompanhamento pré-natal insuficiente ou ausente & 18 & 100 & - & - & - & - \\
\hline \multicolumn{7}{|l|}{ Aspectos de cuidado } \\
\hline Ausência de vínculo & 16 & 88,9 & 02 & 11,1 & - & - \\
\hline Saúde mental materna alterada (ansiedade, depressão e estresse) & 18 & 100 & - & - & - & - \\
\hline $\begin{array}{l}\text { Exposição à violência doméstica (negligência, maus tratos, violência } \\
\text { parental) }\end{array}$ & 18 & 100 & - & - & - & - \\
\hline Desenvolvimento cognitivo dos pais prejudicado & 17 & 94,4 & 01 & 5,6 & - & - \\
\hline Falta de apoio de profissional de saúde & 16 & 88,9 & 02 & 11,1 & - & - \\
\hline Institucionalização & 16 & 88,9 & 02 & 11,1 & - & - \\
\hline $\begin{array}{l}\text { Vivenciar situações estressantes sem ter o apoio suficiente de um cuidador } \\
\text { (adoção, entrada na creche, hospitalização, mudanças familiares)* }\end{array}$ & 17 & 100 & - & - & - & - \\
\hline $\begin{array}{l}\text { Falta de estímulo (ambiente físico inadequado, falta de oportunidade de } \\
\text { brincar, interação inconsistente do cuidador com a criança, entre outras) }\end{array}$ & 16 & 88,9 & 02 & 11,1 & - & - \\
\hline \multicolumn{7}{|l|}{ Condições socioeconômicas } \\
\hline Economicamente desfavorecido (renda familiar insuficiente, desemprego) & 17 & 94,4 & 01 & 5,6 & - & - \\
\hline $\begin{array}{l}\text { Condições sociais desfavoráveis (violência, falta de acesso a rede de apoio, } \\
\text { entre outras) }\end{array}$ & 18 & 100 & - & - & - & - \\
\hline
\end{tabular}

* $\mathrm{N}$ teve valor inferior porque uma pessoa não respondeu o item 
Quanto à definição desse $\mathrm{DE}$, cabe esclarecer que este estudo ocorreu anteriormente à publicação da edição 2015-2017 da NANDA-I ${ }^{(17)}$, na qual é orientado que se utilize o termo "Vulnerabilidade de" para definir um diagnóstico de risco. Apresenta-se aqui a definição que foi avaliada na pesquisa, mas esta será revista para ser submetida à próxima edição da NANDA-I.

Com relação a esse diagnóstico, o grupo focal analisou apenas as alterações sugeridas para os fatores relacionados, que foram as mesmas do diagnóstico anterior, aceitando-as igualmente.

\section{Diagnóstico de enfermagem: “Disposição para desenvolvimento infantil melhorado”}

A Tabela 3 apresenta os resultados da análise do DE “Disposição para desenvolvimento infantil melhorado" pelos juízes.

Tabela 3: Concordância sobre os elementos do diagnóstico Disposição para desenvolvimento infantil melhorado. São Paulo, SP, Brasil, 2014.

\begin{tabular}{|c|c|c|c|c|c|c|}
\hline \multirow[t]{2}{*}{ Componente } & \multicolumn{2}{|c|}{ Concorda } & \multicolumn{2}{|c|}{$\begin{array}{c}\text { Concorda } \\
\text { parcialmente }\end{array}$} & \multicolumn{2}{|c|}{$\begin{array}{c}\text { Não } \\
\text { concorda }\end{array}$} \\
\hline & $\mathbf{N}$ & $\%$ & $\mathbf{N}$ & $\%$ & $\mathbf{N}$ & $\%$ \\
\hline Título: “Disposição para desenvolvimento infantil melhorado" & 11 & 61,1 & 06 & 33,3 & 01 & 5,6 \\
\hline $\begin{array}{l}\text { Definição: Um padrão de cuidado que é suficiente para apoiar/promover o } \\
\text { processo de desenvolvimento da criança e pode ser fortalecido/reforçado }\end{array}$ & 11 & 61,1 & 06 & 33,3 & 01 & 5,6 \\
\hline \multicolumn{7}{|l|}{ Características definidoras } \\
\hline Evidência de vínculo da criança com o cuidador & 18 & 100 & - & - & - & - \\
\hline Necessidades da criança atendidas & 17 & 94,4 & 01 & 5,6 & - & - \\
\hline Ambiente familiar favorável ao desenvolvimento & 17 & 94,4 & 01 & 5,6 & - & - \\
\hline Condições econômicas favoráveis & 15 & 83,3 & 02 & 11,1 & 01 & 5,6 \\
\hline Contexto social adequado & 16 & 88,9 & 02 & 11,1 & - & - \\
\hline Apoio de profissional de saúde & 17 & 94,4 & 01 & 5,6 & - & - \\
\hline Interação com os pais & 14 & 77,8 & 04 & 22,2 & - & - \\
\hline Crescimento adequado & 17 & 94,4 & 01 & 5,6 & - & - \\
\hline Criança amamentada & 14 & 77,8 & 04 & 22,2 & - & - \\
\hline $\begin{array}{l}\text { Apoio dos cuidadores para vivenciar situações de estresse (entrada na } \\
\text { creche, hospitalização, mudanças familiares, etc) }\end{array}$ & 14 & 77,8 & 04 & 22,2 & - & - \\
\hline
\end{tabular}

A proposta de um novo diagnóstico voltado para a promoção do desenvolvimento infantil teve concordância abaixo do valor estabelecido de $80 \%$ em relação ao título e definição, porém, nos comentários, notou-se ser um diagnóstico considerando relevante. As discordâncias foram justificadas por questionamentos aos termos específicos da taxonomia - "disposição", no título, e "um padrão de cuidado", na definição. No grupo focal, o consenso foi de manter o título e definição propostos, uma vez que os termos questionados correspondem à orientação da taxonomia.

Quanto à validação das características definidoras propostas para este diagnóstico, três tiveram índice de concordância inferior a 80\%, porém muito próximo deste valor: "Interação com os pais", "Criança amamentada", "Apoio dos cuidadores para vivenciar situações de estresse (entrada na creche, hospitalização, mudanças familiares, entre outras)". Devido à evidência científica destas características como promotoras do desenvolvimento infantil(18-20), a discussão do grupo focal chegou ao consenso de mantê-las. Três pessoas sugeriram incluir a palavra "favorável" na característica "Interação com os pais" o que foi considerado pertinente na discussão do grupo focal. 


\section{DISCUSSÃO}

O objetivo do estudo foi alcançado, com alto índice de concordância para os diagnósticos propostos. Isto demonstra a adequação dos diagnósticos elaborados com base em estudo consistente da análise de conceito ${ }^{8}$, que permitiu identificar os principais elementos pertinentes ao $\mathrm{DI}$, uma vez que nenhum elemento apresentado para validação foi refutado e também não houve sugestão da inclusão de outros elementos. Nos casos em que não se atingiu o índice de concordância estabelecido, houve poucas discordâncias e maior frequência de concordância parcial, em que foram sugeridas alterações pontuais. Todas as sugestões e comentários apresentados pelos juízes e peritos se referiram à redação e a aspectos próprios da taxonomia da NANDA-(17).

Ainda que o baixo número de juízes participantes possa ser considerado um limite, constituiu-se um grupo qualificado, que atendeu aos critérios de inclusão, afirmando familiaridade com a taxonomia e experiência clínica sobre o fenômeno. Destaca-se que, na época da pesquisa, não havia recomendação da literatura para considerar a experiência clínica nos critérios de seleção de juízes, mas as pesquisadoras optaram por incluir esse critério, por considerá-lo relevante. Corrroborando tal decisão, estudo recente fez uma crítica aos critérios usuais para a seleção dos peritos em pesquisas, propondo considerar a experiência clínica(21).

Quanto à análise dos conteúdos, observou-se que o crescimento prejudicado, não alcançou a concordância estabelecida como fator relacionado ao diagnóstico de atraso no DI, embora tenha alcançado $100 \%$ de concordância como fator de risco. Esse resultado é, de certa forma, surpreendente, e não é possível explicá-lo com os dados da pesquisa, dado que não houve justificativas dos juízes que responderam concordar parcialmente com esse fator. Contudo, isso aponta para a possibilidade de que os participantes dessa etapa da pesquisa ainda não tivessem apreensão aprofundada dos componentes da taxonomia, ainda que tenham afirmado utilizá-la em sua prática, uma vez que um fator de risco presente numa situação de diagnóstico de atraso no DI teria que ser considerado como um fator relacionado a esse diagnóstico.

A incoerência observada nesses resultados acerca do fator relacionado "crescimento inadequado", bem como o estranhamento quanto à composição do diagnóstico de promoção, relaciona-se a um dos desafios da pesquisa sobre diagnósticos. Selecionar os peritos e alcançar o número recomendado na literatura para realizar a validação dos diagnósticos é confirmado como uma das dificuldades dos estudos ${ }^{(14)}$.

Neste estudo, também houve dificuldade de devolutiva dos sujeitos da pesquisa, na etapa de validação por juízes, como se verificou pelo número de respostas. O baixo índice de respostas pode ter se devido ao próprio tema da pesquisa, isto é, diagnósticos de enfermagem. Os profissionais para os quais foi divulgada a pesquisa podem ter decidido não responder por não se sentirem capazes de considerar os aspectos específicos da taxonomia.

Por outro lado, a discussão no grupo focal permitiu a análise mais detalhada dos resultados obtidos com os juízes, o que trouxe contribuições importantes para a proposta final de revisão dos diagnósticos. Confirmou-se que a capacidade interativa e problematizadora do grupo focal como técnica de coleta e análise de dados insere os participantes da pesquisa no contexto das discussões contribuindo, desta forma, para o repensar de concepções e práticas ${ }^{(22)}$ e alcançar maior densidade nos resultados.

As discordâncias com relação ao diagnóstico de promoção de saúde, justificadas por questionamentos aos termos específicos da taxonomia, podem ser devidas ao próprio processo de proposição e refinamento dos termos 
da NANDA-I, que ainda está em construção. Além disso, pode-se considerar que os diagnósticos de promoção são menos usuais, lembrando que, inicialmente, os diagnósticos da NANDA-I eram mais centrados em problemas/distúrbios. Portanto, o estranhamento desses termos não invalida a afirmação dos participantes de terem familiariade com a taxonomia, mas pode evidenciar ainda a utilização mais voltada para as situações de hospitalização e de diagnósticos de problemas.

Considera-se que os diagnósticos de enfermagem precisam ser claros e concisos para conseguirem comunicar os achados e conclusões dos profissionais de enfermagem sobre determinado problema ou situações que o paciente está enfrentando, sendo a referência para orientar as intervenções de enfermagem e medir os resultados de suas ações ${ }^{(23)}$. Assim, a avaliação dos juízes e peritos sobre os diagnósticos de DI possibilitou seu refinamento, deixando-os prontos para o teste clínico.

\section{CONCLUSÃO}

Os resultados deste estudo de validação de conteúdo demonstram que os novos diagnósticos apresentam conteúdos pertinentes ao fenômeno Desenvolvimento Infantil e estão estruturados de acordo com a Taxonomia da NANDA-I. Portanto, estão adequados para serem submetidos à validação clínica para possível incorporação à taxonomia. Isso implicará melhores recursos para os enfermeiros atuarem na atenção integral à saúde da criança.

A combinação de estratégias de pesquisa quanti e qualitativa possibilitou alcançar um grupo mais ampliado de participantes, bem como reflexão e discussão sobre as questões duvidosas, levando à obtenção de resultados consistentes. Pesquisas com diagnósticos de enfermagem são recentes e o uso de diferentes estratégias de pesquisa permite explorar e consolidar este campo de pesquisa.

\section{REFERÊNCIAS}

1. Souza JM, Cruz DALM, Verissimo MLOR. Child Development: New Diagnoses for the NANDA International. Int J Nurs Knowl [Internet]. 2018 [acesso em: 19 jun. 2018];29(2):112-6. Disponível em: https://doi.org/10.1111/2047-3095.12167.

2. Mello DF, Wernet M, Veríssimo MLOR, Tonete VLP. Nursing care in early childhood: contributions from intersubjective recognition. Rev Bras Enferm [Internet]. 2017 [acesso em: 19 jun. 2018];70(2):446-50. Disponível em:

http://dx.doi.org/10.1590/0034-7167-2016-0319.

3. Portaria no 1.130, de 05 de agosto de 2015 (BR) [Internet]. Institui a Política Nacional de Atenção Integral à Saúde da Criança (PNAISC) no âmbito do Sistema Único de Saúde (SUS). Diário Oficial da União. 6 ago. 2015. Disponível em:

http://bvsms.saude.gov.br/bvs/saudelegis/gm/2015/prt1130 0508 2015.html.

4. NANDA-International. Diagnósticos de enfermagem da NANDA: definições e classificações 2009-2011. Garcez RM, tradutora. Porto Alegre: Artmed; 2010.

5. Souza JM, Veríssimo MLOR. Child Development in the NANDA-I and International Classification for Nursing Practices Nursing Classifications. Int J Nurs Knowl [Internet]. 2013 [acesso em: 19 jun. 2018];24(1):44-8. Disponível em:

https://doi.org/10.1111/j.2047-3095.2012.01228.x.

6. Herdman TH. Diagnósticos de enfermagem da NANDA International: definições e classificação, 2012-2014. Garcez RM, tradutora. Porto Alegre: Artmed; 2013.

7. Scroggins LM. The developmental processes for NANDA International Nursing Diagnoses. Int J Nurs Terminol Classif [Internet]. 2008 [acesso em: 19 jun. 2018];19(2):57-64. Disponível em: http://doi.org/10.1111/j.1744-618X.2008.00082.x.

8. Souza JM, Veríssimo MLOR. Child development: analysis of a new concept. Rev Lat Am Enfermagem [Internet]. 2015 [acesso em: 19 jun. 2018];23(6):1097-104. Disponível em: http://dx.doi.org/10.1590/0104-1169.0462.2654.

9. Talmelli LFS, Gratão ACM, Rodrigues RAP. Modelo Híbrido para desenvolvimento de conceito em Enfermagem utilizando Revisão Integrativa e Análise de Conteúdo. Saúde \& Transformação Social [Internet]. 2016 [acesso em: 19 jun. 2018];7(1):9-15. Disponível em: http://incubadora.periodicos.ufsc.br/index.php/saudeetransformacao/article/view/4118.

10. Bronfenbrenner U. Bioecologia do desenvolvimento humano. Tornando os seres humanos mais humanos. Porto Alegre:

Artmed; 2011. 
11. Resolução № 466 do Conselho Nacional de Saúde, de 12 de dezembro de 2012 (BR) [Internet]. Aprova as diretrizes e normas regulamentadoras de pesquisas envolvendo seres humanos. Diário Oficial da União. 12 dez 2012 [acesso em: 19 jun. 2018]. Disponível em: http://bvsms.saude.gov.br/bvs/saudelegis/cns/2013/res0466 1212 2012.html.

12. Paranhos R, Figueiredo Filho DB, Rocha EC, Sival Junior JA, Freitas D. Uma introdução aos métodos mistos. Sociologias [Internet]. 2016 [acesso em: 19 jun. 2018];18(42):384-411. Disponível em: http://dx.doi.org/10.1590/15174522-018004221. 13. Alexandre NMC, Coluci MZO. Validade de conteúdo nos processos de construção e adaptação de instrumentos de medidas. Cien Saude Colet [Internet]. 2011 [acesso em: 19 jun. 2018];16(7):3061-3068. Disponível em: http://dx.doi.org/10.1590/S141381232011000800006.

14. Lopes MVO, Silva VM, Araujo TL. Validação de diagnósticos de enfermagem: desafios e alternativas. Rev Bras Enferm [Internet]. 2013 [acesso em: 19 jun. 2018];66(5):649-55. Disponível em: http://dx.doi.org/10.1590/S0034-71672013000500002.

15. Nash A, Dunn M, Asztalos E, Corey M, Mulvihill-Jory B, O'Connor DL. Pattern of growth of very low birth weight preterm infants, assessed using the WHO Growth Standards, is associated with neurodevelopment. Appl Physiol Nutr Metab [Internet]. 2011 [acesso em: 19 jun. 2018];36(4):562-9. Disponível em: http://doi.org/10.1139/h11-059.

16. Park H, Bothe D, Holsinger E, Kirchner HL, Olness K, Mandalakas A. The impact of nutritional status and longitudinal recovery of motor and cognitive milestones in internationally adopted children. Int J Environ Res Public Health [Internet]. 2011 [acesso em: 19 jun. 2018];8(1):105-16. Disponível em: http://doi.org/10.3390/ijerph8010105.

17. Herdman TH, Kamitsuru S. NANDA International Nursing Diagnoses: Definitions and Classification, 2015-2017. Oxford (UK): Wiley-Blackwell; 2014.

18. Belsky J, de Haan M. Annual Research Review: Parenting and children's brain development: the end of the beginning. J Child Psychol Psychiatry [Internet]. 2011 [acesso em: 19 jun. 2018];52(4):409-28. Disponível em: http://doi.org/10.1111/i.1469-

7610.2010.02281.x.

19. Krol KM, Rajhans P, Missana M, Grossmann T. Duration of exclusive breastfeeding is associated with differences in infants' brain responses to emotional body expressions. Front Behav Neurosci [Internet]. 2015 [acesso em: 19 jun. 2018];8:459. Disponível em: http://doi.org/10.3389/fnbeh.2014.00459.

20. World Health Organization. Developmental difficulties in early childhood: prevention, early identification, assessment and intervention in low- and middle-income countries: a review. 2012 [Internet]. Genebra (SU): World Health Organization; 2012 [acesso em: 19 jun. 2018]. Disponível em:

http://www.who.int/maternal child adolescent/documents/development difficulties early childhood/en/.

21. Guimarães HCQCP, Pena SB, Lopes JL, Lopes CT, Barros ALBL. Experts for Validation Studies in Nursing: New Proposal and Selection Criteria. Int J Nurs Knowl [Internet]. 2016 [acesso em: 19 jun. 2018];27(3):130-5. Disponível em: http://doi.org/10.1111/2047-3095.12089.

22. Backes DS, Colomé JS, Erdmann RH, Lunardi VL. Grupo focal como técnica de coleta e análise de dados em pesquisas qualitativas. O Mundo da Saúde [Internet]. 2011 [acesso em: 19 jun. 2018];35(4):438-442. Disponível em: http://doi.org/10.15343/0104-7809.2011354438442.

23. Carvalho EC, Cruz DALM, Herdman TH. Contribuição das linguagens padronizadas para a produção do conhecimento, raciocínio clínico e prática clínica da Enfermagem. Rev Bras Enferm [Internet]. 2013 [acesso em: 19 jun. 2018];66(spe):134-41. Disponível em: http://dx.doi.org/10.1590/S0034-71672013000700017. 